The Astrophysical Journal, 412:301-311, 1993 July 20

(C) 1993. The American Astronomical Society. All rights reserved. Printed in U.S.A.

\title{
MUON ASTRONOMY WITH THE MACRO DETECTOR
}

S. Ahlen, ${ }^{1}$ M. Ambrosio, ${ }^{2}$ R. Antolini,${ }^{3}$ G. Auriemma,,${ }^{4,5}$ A. Baldini,${ }^{6}$ B. Bam,${ }^{3}$ G. Barbarino, ${ }^{2}$ B. Barish, ${ }^{7}$ G. Battistoni, ${ }^{8,9}$ R. Bellotti,${ }^{10}$ C. Bemporad, ${ }^{6}$ P. Bernardini, ${ }^{11}$ H. Bilokon, ${ }^{8}$ V. Bisi ${ }^{12}$ C. Bloise, ${ }^{8}$ S. Bussino, ${ }^{4}$ F. Cafagna, ${ }^{10}$ M. Calicchio, ${ }^{10}$ P. Campana, ${ }^{8}$ D. Campana, ${ }^{2}$ M. Carboni ${ }^{8}$ S. CeCchini ${ }^{3,13}$ F. Cei, ${ }^{6}$ V. Chiarella,${ }^{8}$ C. Chiera, ${ }^{4}$ A. Cobis, ${ }^{8}$ R. Cormack, ${ }^{1}$ A. Corona,${ }^{4}$ S. Coutu, ${ }^{7}$ G. DeCataldo, ${ }^{10}$ H. Dekhissi, ${ }^{3,14}$ C. DeMarzo, ${ }^{10}$ M. De Vincenzi ${ }^{4}$ A. Di Credico, ${ }^{15}$ E. Diehl, ${ }^{16}$ O. Erriquez, ${ }^{10}$ C. Favuzzi ${ }^{10}$ D. Ficenec, ${ }^{1,17}$ C. Forti, ${ }^{8}$ L. Foti, ${ }^{4}$ P. Fusco, ${ }^{10}$ G. Giacomelli, ${ }^{3}$ G. Giannini,,${ }^{6,18}$ N. Giglietto, ${ }^{10}$ P. Giubellino, ${ }^{12}$ M. Grassi, ${ }^{6}$ P. Green, ${ }^{19}$ A. Grillo, ${ }^{8}$ F. Guarino, ${ }^{2}$ C. Gustavino, ${ }^{20}$ A. Habig, ${ }^{21}$ R. Heinz, ${ }^{21}$ J. Hong, ${ }^{7}$ E. IAROCCI, ${ }^{8,22}$ E. KatSavounidis, ${ }^{7}$ E. Kearns, ${ }^{1}$ S. Klein, ${ }^{1,23}$ S. Kyriazopoulou, ${ }^{7}$ E. Lamanna, ${ }^{4}$ C. Lane, ${ }^{24}$ C. LeE, ${ }^{16}$ D. Levin,,${ }^{16}$ P. Lipari, ${ }^{4}$ G. LiU, ${ }^{7}$ R. Liu, ${ }^{7}$ M. Longo, ${ }^{16}$ G. Ludlam, ${ }^{1}$ G. Mancarella,${ }^{11}$ G. Mandrioli,${ }^{3}$ A. Margiotta-Neri, ${ }^{3}$ A. Marin, ${ }^{1}$ A. Marini ${ }^{8}{ }^{8}$ D. Martello, ${ }^{11}$ G. Martellotti, ${ }^{4}$ A. Marzari Chiesa, ${ }^{12}$ M. Masera, ${ }^{12}$ P. Matteuzzi, ${ }^{3}$ D. Michael, ${ }^{7}$ J. L. Miller, ${ }^{21}$ P. Monacelli, ${ }^{15}$ M. Monteno, ${ }^{12}$ S. L. Mufson, ${ }^{21}$ J. Musser, ${ }^{21}$ S. Nutter, ${ }^{21,16}$ C. Okada, ${ }^{1}$ G. Osteria, ${ }^{2}$ O. Palamara,${ }^{11}$ S. Parlati,${ }^{20}$ V. Patera,${ }^{8}$ L. PatriziI ${ }^{3}$ R. Pazzi, ${ }^{6}$ C. Peck, ${ }^{7}$ J. Petrakis, ${ }^{25}$ S. Petrera ${ }^{11}$ N. Pignatano, ${ }^{7}$ P. Pistilli, ${ }^{11}$ F. Predieri, ${ }^{3}$ L. Ramello, ${ }^{12}$ J. Reynoldson, ${ }^{20}$ F. Ronga, ${ }^{8}$ G. Rosa ${ }^{4}$ C. Satriano ${ }^{4,5}$ L. Satta, ${ }^{8,22}$ E. Scapparone, ${ }^{3}$ K. Scholberg, ${ }^{7}$ A. Sciubba,${ }^{4,22}$ P. Serra lugaresi, ${ }^{3}$ M. Severi, ${ }^{4}$ M. Sitta, ${ }^{12}$ P. Spinelli, ${ }^{10}$ M. SpinetTi,${ }^{8}$ M. Spurio, ${ }^{3}$ J. STeele, ${ }^{7}$ R. Steinberg, ${ }^{24}$ J. Stone, ${ }^{1}$ L. Sulak, ${ }^{1}$ A. Surdo, ${ }^{11}$ G. Tarlé, ${ }^{16}$ V. Togo, ${ }^{3}$ V. Valente, ${ }^{8}$ G. Verdone ${ }^{4,5}$ C. WALTER, ${ }^{7}$ R. WeBb, ${ }^{26}$ AND W. Worstell ${ }^{1}$ (The MACRO Collaboration)

Received 1992 October 26; accepted 1993 January 29

\section{ABSTRACT}

We have analyzed a sample of $1.8 \times 10^{6}$ muons with $E_{\mu} \gtrsim 1.3 \mathrm{TeV}$ collected by the first and second supermodules of the MACRO detector at Gran Sasso during the period 1989 February to 1991 February. We have searched for an excess of muons of celestial origin over cosmic-ray background. Our search for steady sources in solid angle bins of $\Delta \Omega \approx 3.0 \times 3.0$ in the declination range $-5^{\circ} \leq \delta \leq 90^{\circ}$ was negative; the upper limit with $95 \%$ C.L. to the muon flux at mid-latitudes $\left(\delta \sim 45^{\circ}\right)$ is $J_{\mu}^{\text {steady }} \lesssim 2 \times 10^{-12} \mathrm{~cm}^{-2} \mathrm{~s}^{-1}$. In addition, we have searched for muon excesses modulated by the orbital period of selected X-ray sources, including Cyg X3, Cyg X-1, Her X-1, 4U 0115+63, and 4U $1907+09$. For Cyg X-3 we obtained an upper limit with 95\% C.L. of $J_{\mu}^{\text {mod }} \leq 8.8 \times 10^{-13} \mathrm{~cm}^{-2} \mathrm{~s}^{-1}$. Further, we have no evidence of any bursting activity from Cyg X-3, during our search period, which includes the radio outburst of 1991 January.

Subject headings: elementary particles - gamma rays: observations

\section{INTRODUCTION}

During the period 1975-1985 there appeared a growing body of experimental evidence for the existence of UHE $\gamma$-rays from the direction of point sources like the bright X-ray binary Cyg X-3. For these sources, the integral $\gamma$-ray flux at TeV energies is like that measured for Cyg X-3, $N\left(>E_{\gamma}\right)=K E_{\gamma}^{-\alpha}$, where

\footnotetext{
1 Physics Department, Boston University, Boston, MA 02215.

2 Dipartimento di Fisica dell'Universitá di Napoli and INFN, Napoli, 80125 , Italy.

${ }^{3}$ Dipartimento di Fisica dell'Universitá di Bologna and INFN, Bologna, 40126, Italy.

${ }^{4}$ Dipartimento di Fisica dell'Universitá di Roma and INFN, Roma, 00185, Italy.

${ }^{5}$ Also Universitá della Basilicata, Potenza, 85100, Italy.

${ }^{6}$ Dipartimento di Fisica dell'Universitá di Pisa and INFN, Pisa, 56010, Italy.

${ }^{7}$ California Institute of Technology, Pasadena, CA 91125.

${ }^{8}$ Laboratori Nazionali di Frascati dell'INFN, Frascati (Roma), 00044, Italy.

9 Now at INFN Sezione di Milano, Milano, 201333, Italy.

${ }^{10}$ Dipartimento di Fisica dell'Universitá di Bari and INFN, Bari, 70126, Italy.

${ }_{11}$ Dipartimento di Fisica dell'Universitá di Lecce and INFN, Lecce, 73100, Italy.

${ }_{12}$ Dipartimento di Fisica dell'Universitá di Torino and INFN, Torino, 10125 , Italy.

${ }^{13}$ Also Istituto TESRE/CNR, Bologna, Italy.
}

$\alpha=1.1 \pm 0.1$ (Nagle, Gaisser, \& Protheroe 1988). One striking feature of the Cyg X-3 signal in particular was that the showers were not "muon poor," as expected for electromagnetic showers (Auriemma, Bilokon, \& Grillo 1984; Stanev, Vankov, \& Halzen 1985; and Berezinsky et al. 1988).

In addition, two underground experiments reported excesses of $\mathrm{TeV}$ muons pointing to the direction of Cyg X-3: Soudan 1

\footnotetext{
${ }^{14}$ Also Department of Physics, University Mohammed Ier, Oujda, Morocco.

${ }_{15}$ Dipartimento di Fisica dell'Universitá dell'Aquila and INFN, L'Aquila, 67100 , Italy.

16 Department of Physics, University of Michigan, Ann Arbor, MI 48109.

${ }^{17}$ Now at Physics Department, Washington University, St. Louis, MO 63130.

18 Also Universitá di Trieste and INFN, Trieste, 34100, Italy.

19 Sandia National Laboratory, Albuquerque, NM 87185.

${ }^{20}$ Laboratori Nazionali del Gran Sasso dell'INFN, Assergi (L'Aquila), 67010 , Italy.

${ }^{21}$ Departments of Physics and of Astronomy, Indiana University, Bloomington, IN 47405.

${ }_{22}$ Also Dipartimento di Energetica, Universitá di Roma, Roma, 00185, Italy.

${ }^{23}$ Now at Department of Physics, University of California, Santa Cruz, CA 95064

${ }^{24}$ Department of Physics, Drexel University, Philadelphia, PA 19104.

25 Bartol Research Institute, University of Delaware, Newark, DE 19716.

${ }^{26}$ Physics Department, Texas A\&M University, College Station, TX 77843.
} 
(Marshak et al. 1985a, b) and NUSEX (Battistoni et al. 1985). The most compelling evidence for the association of the muons with Cyg X-3 was the modulation of the muon arrival time with the $4.8 \mathrm{hr}$ orbital period of the source.

Since 1986 further experiments have only been able to place upper limits on the UHE $\gamma$-ray flux from any point source (Weekes 1991). All sources previously reported, for example, Cyg X-3, Her X-1, and 1E 2259+59, have not again been positively identified. Further, investigations with the underground detectors Kamiokande (Oyama et al. 1986), Fréjus (Berger et al. 1986), IMB (Bionta et al. 1987), and Homestake (Corbato 1989), as well as early studies with MACRO (Petrakis 1990), failed to detect any significant muon excess from any source. During this period, only one positive underground muon signal has been reported. The Soudan 2 collaboration reported a burst of muons from Cyg X-3 with a flux of $\sim 7.5 \times 10^{-10}$ $\mathrm{cm}^{-2} \mathrm{~s}^{-1}$ at $2090 \mathrm{~m}$ of water equivalent (m.w.e.), unmodulated by the X-ray period, during a large radio flare in 1991 January (Thomson et al. 1991).

The continuing interest in the detection of muons from cosmic sources comes from the fact that there is no simple explanation within the framework of the standard model of particle physics for such an observation. For muons to preserve source directionality and phase, the primary interacting particle must be low mass, long-lived, and neutral. The most likely neutral primary is a $\gamma$-ray since the zenith angle distribution of the muons seemingly rules out neutrinos (see, e.g., Ruddick 1985, 1986; Berezinsky, Ellis, \& Ioffe 1986; Collins \& Olness 1987). For a $\gamma$-ray primary to produce a muon above the MACRO threshold, it must have an energy $E_{\gamma} \geq 3 \mathrm{TeV}$. Only two sources, the Crab Nebula (Vacanti et al. 1991; Baillon et al. 1991) and Mrk 421 (Punch et al. 1992), are known to emit photons exceeding this limit. Using the results of Berezinsky et al. (1988), the expected flux of muons from the Crab Nebula $(\alpha=1.4)$ is $J_{\mu}\left(E_{\mu}>3 \mathrm{TeV}\right) \approx 10^{-15} \mathrm{~cm}^{-2} \mathrm{~s}^{-1}$, a flux well below the sensitivity of the present search. The situation concerning Cyg X-3 is of course more controversial. However, using the upper limit with $95 \%$ confidence (C. L.) given by O'Flaherty et al. (1991), $J_{\gamma}(>1 \mathrm{TeV}) \leq 3.9 \times 10^{-11} \mathrm{~cm}^{-2} \mathrm{~s}^{-1}$, the limit to the Cyg X-3 muon flux $(\alpha=1.1)$ is $\lesssim 10^{-14} \mathrm{~cm}^{-2}$ $\mathrm{s}^{-1}$. This limit is beyond the sensitivity of the present search, although it could be reached eventually with the full MACRO detector running for several years.

Consequently, the search for muon emission from point sources in the present investigation is a search for exotic processes. Perhaps there are sources that produce unknown, longlived, neutral particles with typical hadronic interactions (see, e.g., Auriemma, Maiani, \& Petrarca 1985; Baym et al. 1985). Or perhaps $\gamma$-ray interactions in the atmosphere at extreme energies involve unexpected processes above $\sim 1 \mathrm{TeV}$, as suggested by Drees \& Halzen (1988) and Yock (1991). Another intriguing possibility is that neutrinos contain strongly interacting preons at a mass scale of $\sim 1 \mathrm{TeV}$ (Domokos \& Nussinov 1987).

In this paper we reconstruct the direction of the muons seen by MACRO to survey the sky for steady pointlike sources of muon excesses over the cosmic-ray background in the declination range $-5^{\circ} \leq \delta \leq 90^{\circ}$. Throughout these investigations we make the assumption that the intrinsic angular spread of the muons (or of the primaries) is small compared with the spread introduced by multiple Coulomb scattering and other electromagnetic processes in the rock. In addition, we have made a more sensitive search for a muon signal from several candidate UHE emitters by using a known periodicity to improve signal-to-noise ratio. Finally, we have searched our list of candidate point sources for bursting behavior similar to that reported for Cyg X-3 during its 1991 January radio flare (Thomson et al. 1991).

\section{THE MACRO EXPERIMENT}

The MACRO (Monopole, Astrophysics, Cosmic Ray Observatory) detector is a large-area, underground experiment designed to search for rare components of cosmic radiation (monopoles, nuclearities, etc.); to search for high-energy neutrinos and muons from cosmic point sources; to detect lowenergy neutrinos from stellar collapse; and to acquire a large sample of multiple muon events from cosmic-ray showers for the investigation of the primary cosmic-ray composition above the "knee."

MACRO is located in Hall B of the Gran Sasso National Laboratory (LNGS) at latitude $42^{\circ} 27^{\prime} \mathrm{N}$, longitude $13^{\circ} 34^{\prime} \mathrm{E}$, at average depth of 3800 m.w.e., and at $963 \mathrm{~m}$ above sea level. When completed, the MACRO detector will have dimensions $72 \mathrm{~m} \times 12 \mathrm{~m} \times 10 \mathrm{~m}$, providing an acceptance for isotropic particle fluxes of $A_{\text {eff }} \Omega \sim 10,000 \mathrm{~m}^{2}$ sr.

The first supermodule of the MACRO detector is fully described in Ahlen et al. (1993). The main components are a streamer tube system, a scintillator system, and track-etch detector. The streamer tube system is primarily designed for accurate tracking of muons and slow monopoles. The functions of the scintillator system are to make precise timing measurements of through-going muons for the discrimination of upward from downward trajectories, to record the waveforms of muons and monopoles, and to detect low-energy neutrinos from stellar collapse. The purpose of the track etch system is to aid in the identification of relatively fast monopoles. The general layout of the full MACRO detector is shown in Figure 1.

The analysis reported in this paper depends primarily on the streamer tube tracking system of the lower deck of the first two supermodules. Each supermodule consists of 10 horizontal planes of streamer tubes operating in the limited streamer mode; in addition there are six vertical planes on each exterior side. The spacing between the central streamer planes is partially filled with rock corresponding to $\approx 60 \mathrm{~g} \mathrm{~cm}^{-2}$; the threshold for a $\mu$ traversing the apparatus in the vertical direction is $\sim 2 \mathrm{GeV}$. Each plane of streamer tubes is read out by wires and pick-up strips at a stereo angle of 26.5 which allow the reconstruction of the hit position within $\sigma_{x}+\sigma_{y}=$ $1.4 \times 1.1 \mathrm{~cm}^{2}$. In the track reconstruction procedure, a search is first made for a set of aligned points. A linear fit to these selected points is then performed, and the track parameters are calculated.

The statistical uncertainty in the direction of reconstructed tracks of the first MACRO supermodule has been experimentally determined by measuring the space angle between the tracks of double muon events. The distribution of these angles is shown in Figure 2. In this distribution approximately $68 \%$ of the events have a space angle $\leq 1.4$, which implies a statistical uncertainty in pointing $1.4 / 2^{1 / 2} \approx 1^{\circ}(1 \sigma)$. This uncertainty is a convolution of several factors. The main contribution is due to multiple Coulomb scattering as the muons traverse the rock overburden. In addition, there is the contribution due to the intrinsic resolution of the detector, which corresponds to an uncertainty of $0.48(1 \sigma)$ (Ahlen et al. 1993). The systematic errors in the absolute pointing are estimated to be $\lesssim 0^{\circ} 25$, based on a survey from geographical references outside the tunnel. 


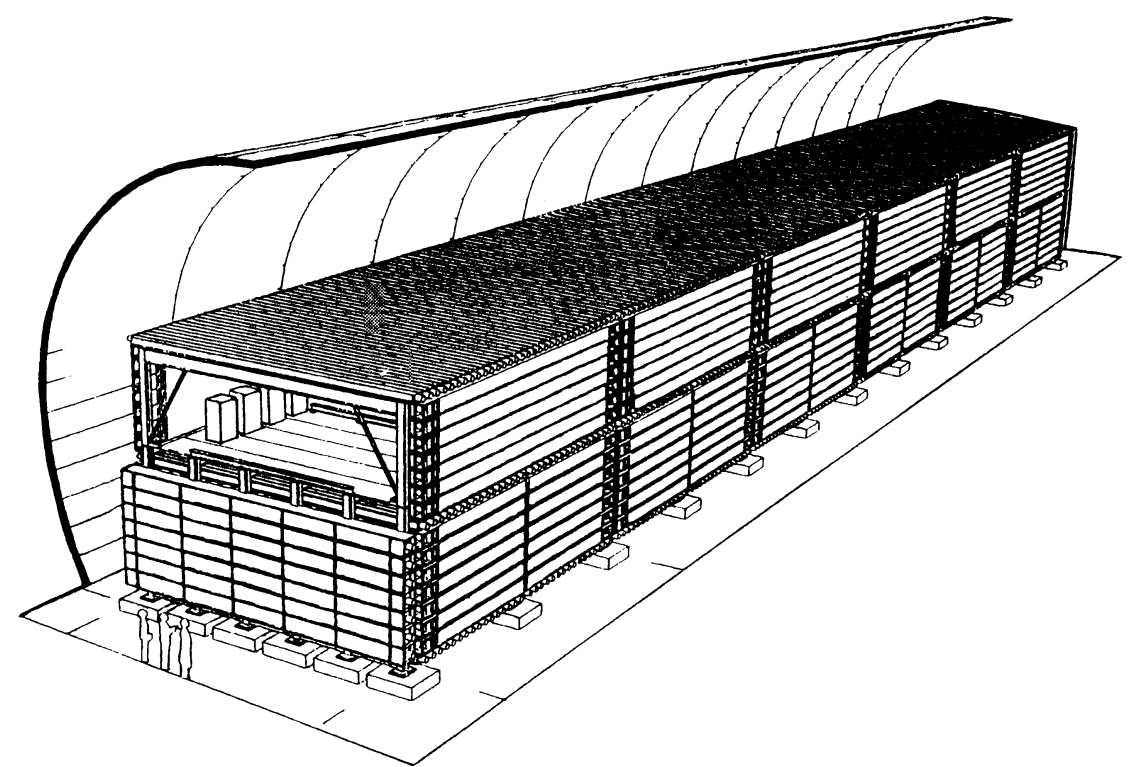

Fig. 1.-General layout of the full MACRO detector in Hall B of the Gran Sasso Laboratory

For the muon astronomy studies reported here, we have conservatively chosen 1.5 as the radius of the search window. This window would contain $81 \%$ of the muons originating from a point source.

\section{DATA SELECTION}

The muon data reported here were collected by the first MACRO supermodule $(12 \mathrm{~m} \times 12 \mathrm{~m} \times 4.8 \mathrm{~m})$ during two extended periods, and by the first and second MACRO supermodules $(24 \mathrm{~m} \times 12 \mathrm{~m} \times 4.8 \mathrm{~m})$ during a third. For each of these three data sets, the dates and the number of active supermodules are given in Table 1 .

The muon detection efficiency is the product of the trigger efficiency, $\epsilon_{t}$, and the track reconstruction efficiency $\epsilon_{r}$. In our apparatus, $\epsilon_{t}>99 \%$ due to multiple triggering schemes. However, the track reconstruction efficiency differs more sig-

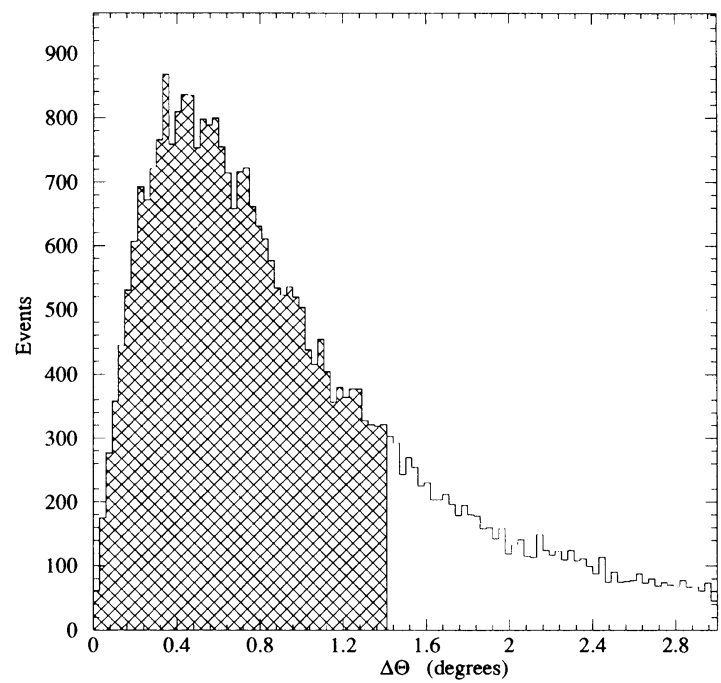

FIG. 2.-Distribution of the the space angle $\Delta \Theta$ between reconstructed tracks in double muon events. Over $68 \%$ of the events fall within the crosshatched region. nificantly from 1 because of its dependence on factors like the efficiency of the streamer tubes and the requirements of the reconstruction procedure (viz., $\geq 4$ of 10 planes hit). We have evaluated $\epsilon_{r}$ on a run-by-run basis by selecting clean muon events using two separate scintillator triggers. We used scintillator information alone for this sample of muon events in order to keep it free from the bias of using streamer tube information to define the efficiency of the streamer tube system. We have investigated the possibility that a hot scintillator box could generate spurious muon events as selected by our criteria. Our studies demonstrate that such spurious muon events are too few to affect significantly our computation of the efficiency.

This sample shows that $\epsilon_{r}$ has a Gaussian distribution with a mean of $\bar{\epsilon}_{r} \approx 0.81$. The factor that reduces the track reconstruction efficiency most significantly is its dependence on track length, in the sense that shorter tracks have a lower probability of being reconstructed. In particular, corner clippers are likely to trigger the apparatus, although they are unlikely to give a reconstructed muon track. The $\epsilon_{r}$ quoted above is an average over all track lengths and should be regarded as a conservative estimate.

During data taking period 3 , no scintillator trigger was in operation on the second supermodule (SM2). We have estimated the muon detection efficiency in SM2 during this time to be that of SM1, scaled by the ratio of the number of reconstructed tracks in SM1 and SM2.

For each data set, we list in Table 2 the number of events with reconstructed streamer tube tracks. We then applied two general types of cuts to these events, those which eliminate entire runs and those which target specific types of events within a run. The following types of runs were cut: (1) Runs

TABLE 1

Parameters of Muon Data Sets

\begin{tabular}{ccc}
\hline \hline Set Number & \multicolumn{1}{c}{ Period of Data Taking } & SMs Active \\
\hline $1 \ldots \ldots \ldots \ldots \ldots \ldots \ldots$ & 1989 Feb 27 -1989 May 30 & 1 \\
$2 \ldots \ldots \ldots \ldots \ldots \ldots \ldots$ & 1989 Nov 11-1990 May 10 & 1 \\
$3 \ldots \ldots \ldots \ldots \ldots \ldots \ldots$ & 1990 May 10-1991 Feb 5 & 1,2 \\
\hline
\end{tabular}


TABLE 2

Events Lost to CuTS

\begin{tabular}{|c|c|c|c|c|c|}
\hline \multirow[b]{2}{*}{ SET Number } & \multirow{2}{*}{$\begin{array}{l}\text { NuMbER OF } \\
\text { EVENTS }\end{array}$} & \multicolumn{2}{|c|}{ EVENTS LOST TO } & \multirow{2}{*}{$\begin{array}{c}\text { NUMBER OF } \\
\text { EVENTS ANALYZED }\end{array}$} & \multirow{2}{*}{$\begin{array}{c}\text { Exposure TIME } \\
(\mathrm{hr})\end{array}$} \\
\hline & & Run Cuts & Event Cuts & & \\
\hline $1 \ldots \ldots \ldots \ldots$ & 249795 & 2154 & 3649 & 244333 & 1942.87 \\
\hline $2 \ldots \ldots \ldots \ldots$ & 385228 & 9287 & 13321 & 365148 & 3072.05 \\
\hline $3 \ldots \ldots \ldots \ldots$ & 1388533 & 53331 & 27281 & 1235321 & 5274.90 \\
\hline Total ........ & 2023556 & 64772 & 44251 & 1844802 & 10289.82 \\
\hline
\end{tabular}

with low efficiencies were cut. The cutoff was set at $3 \sigma$ below $\bar{\epsilon}_{r}$ for that data set as determined by fitting a Gaussian function to the efficiency distribution. (2) We have excluded runs with peculiar rates, specifically those with an event rate less than $10 / \mathrm{hr} /$ supermodule (usually very short runs), or an event rate larger than $250 / \mathrm{hr} /$ supermodule (short runs or runs with peculiar streamer tube activity). (3) Runs were cut for spurious data acquisition behavior. We have applied the following event cuts to the data: (1) Events with zenith angle greater than $72^{\circ}$ were excluded. (2) Events were excluded if the multiplicity in the strip view and the multiplicity in the wire view differed by more than 2. (3) Events were excluded when tagged with an incorrect UTC time. The number of events excluded due to run and event cuts is listed in Table 2 . This is followed by a column that gives for each data set the number of events analyzed. A total of approximately $1.8 \times 10^{6}$ events survive the cuts. The final column of Table 2 gives the exposure time, defined as the time the detector was actively collecting these data.

The zenith angle and azimuthal angle distributions for all the events in the local reference frame are shown in Figure 3. The shapes of these distributions reflect the mountain overburden. In Figure 4 we plot the right ascension and sin declination $(\sin \delta$ ) distribution for these events. The structure seen in the right ascension distribution is primarily due to the effects of detector downtime.

\section{SEARCH CRITERION}

The muons seen in MACRO are primarily the result of the interaction of charged cosmic-ray primaries $(E>10 \mathrm{TeV})$ with the atmosphere, although single muons can result from pri- maries of lower energy. Above the Earth's atmosphere, these cosmic-ray primaries are isotropically distributed due to propagation effects through the Galactic magnetic field. At the surface of the Earth, the distribution of these muons integrated over long times is expected to be highly isotropic in right ascension, but to have a declination distribution that depends on the observer's latitude (through the zenith angle dependence of muon production). Underground at MACRO, this muon distribution will remain isotropic in right ascension, but its declination distribution will be modified by the known rock overburden. A search for astrophysical sources of muons, therefore, can be made by looking for a statistically significant excess of muons from a particular direction with respect to the expected number of muons from that direction.

The distribution of muons actually observed at MACRO from an isotropic primary flux, however, will match this expected distribution only as long as there are no gaps in the data taking and the detector remains perfectly efficient. To account for the time gaps that do occur and the changing detector efficiency, we have computed the expected muon distribution on a run-by-run basis by Monte Carlo simulation. In the following we will use the term "background" to designate the expected number of muons computed in this way.

The simulation is based on the fact that for an isotropic distribution of cosmic-ray primaries, the muon arrival times and directions are uncorrelated and therefore can be chosen independently. The simulated arrival times for each run were initialized with the observed time for the first event in that run. Event arrival times were simulated by using the mean time between events for each run to choose exponential deviates, $\Delta t$,

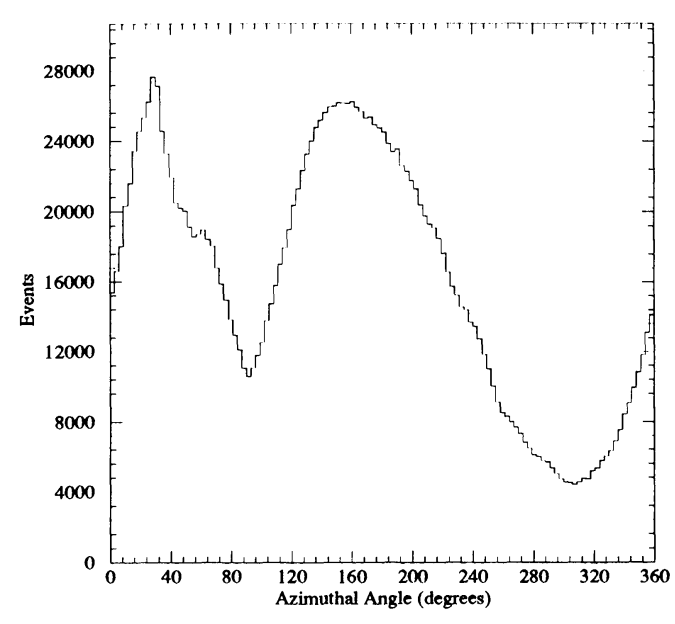

FIG. $3 a$

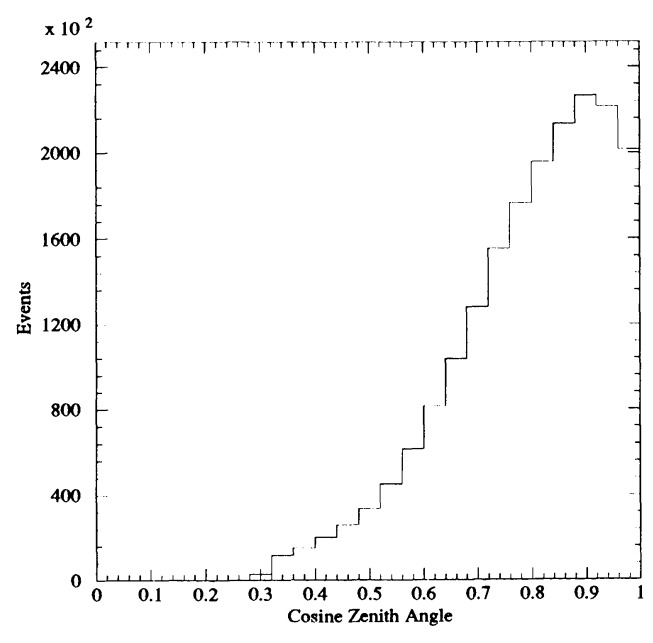

FIG. $3 b$

FIG. 3.-(a) Azimuthal angle distribution for the total muon sample. (b) Cosine zenith angle distribution for the total muon sample. 


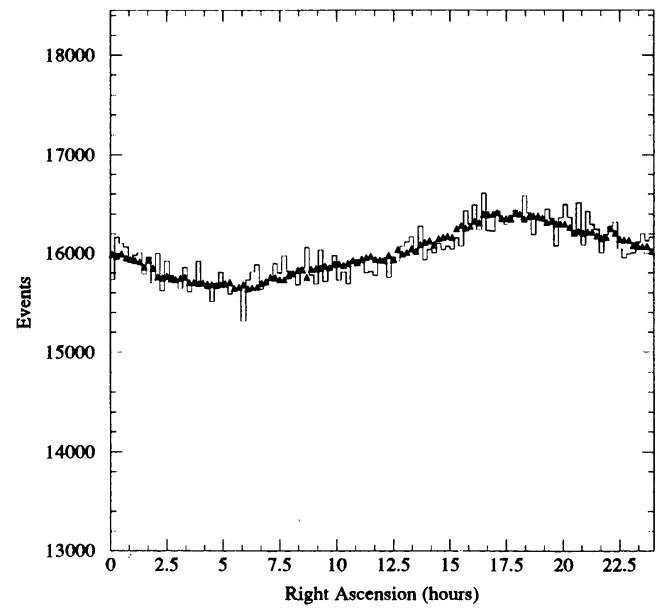

FIG. $4 a$

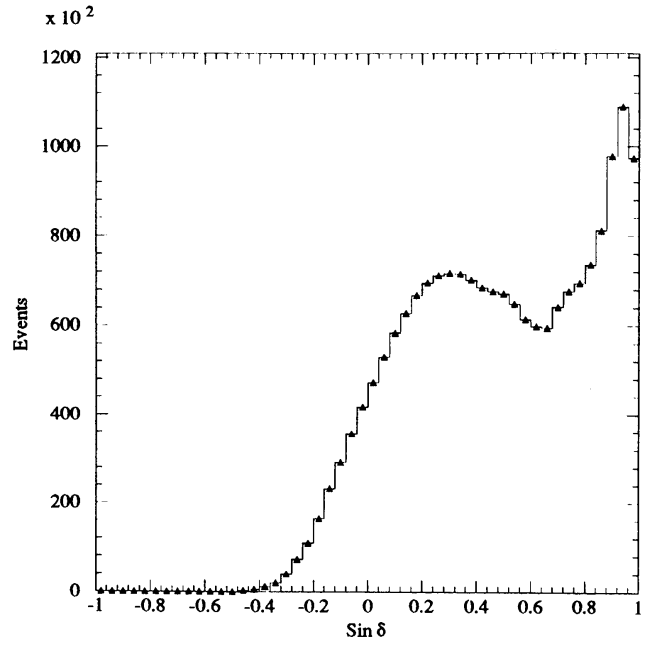

FIG. $4 b$

Fig. 4. - (a) Right ascension distribution for the total muon sample. The average of 25 Monte Carlo simulations is superposed. (b) sin $\delta$ distribution for the total muon sample. The average of 25 Monte Carlo simulations is superposed.

and then computing $t_{i+1}=t_{i}+\Delta t$. The dead time is sufficiently short $(\sim 50 \mathrm{~ms})$ compared with the mean time between events $(>10 \mathrm{~s})$ that it could be ignored in these computations. This method successfully reproduced the distribution of time differences between events (Ahlen et al. 1992), particularly important consideration in the search for periodicities. Event positions were then chosen from the observed two-dimensional distribution of zenith and azimuthal angles. We combined the simulated position and time for each event to calculate the expected right ascension and declination.

In Figures $4 a$ and $4 b$, the right ascension and $\sin \delta$ distributions generated by an average of 25 Monte Carlo simulations are shown as triangles superposed onto the data distributions. We use the average of 25 Monte Carlos since fluctuations in the simulated right ascension distribution $(\sigma \approx 25$ events) are then small compared with fluctuations in the data $(\sigma \approx 125$ events). The background simulations reproduce the structure in the right ascension quite well because the gaps in continuous running are accounted for explicitly in the Monte Carlo.

\section{ALL-SKY SURVEY}

We have used the right ascension and declination distributions to search the sky accessible to MACRO for statistically significant sources of muon excess. As a first step the muons were sorted into bins of equal solid angle, $\Delta \Omega=2.1 \times 10^{-3}$ $(\Delta \alpha=3.0, \Delta \sin \delta=0.04)$. These bins have approximately the same $\Delta \Omega$ as a cone of half-angle 1.5 .

The deviation from the mean for each solid angle bin was computed using the Gaussian statistic $\left(n_{\text {obs }}-n_{\text {exp }}\right) /\left(n_{\text {exp }}\right)^{1 / 2}$, where $n_{\text {obs }}$ is the observed number of events in the bin and $n_{\exp }$ is the number of events expected from background. Figure $5 a$ shows the distribution of these deviations for the 3960 bins in which the number of events is $\geq 16$. Bins with small numbers of events were excluded because the Gaussian approximation to the deviation is not valid for them. Superposed onto this distribution of deviations is the best-fitting Gaussian, for which $\chi^{2} / \mathrm{DoF}=87.7 / 68$. The mean of the Gaussian is near zero $\left(-1.6 \times 10^{-2}\right)$ and $\sigma$ is 1.0 , as expected for a distribution of

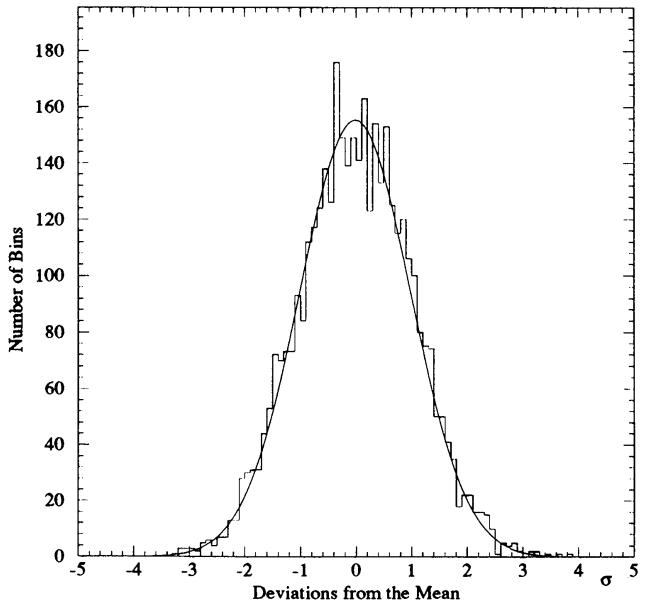

FIG. $5 a$

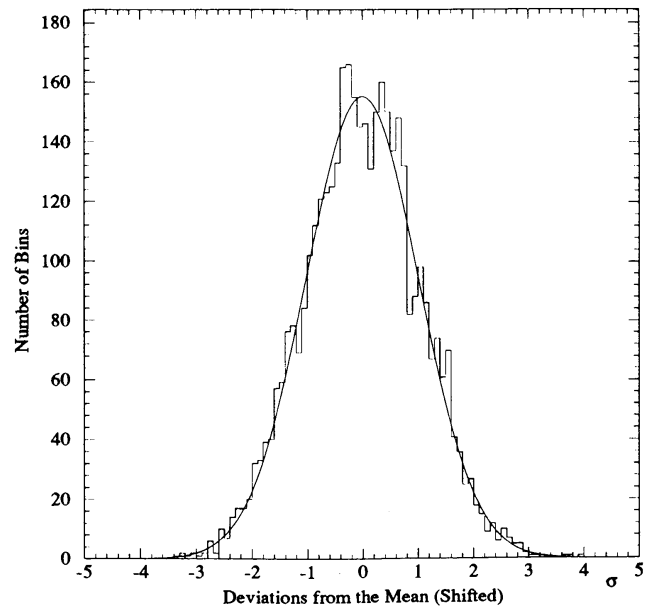

FIG. $5 b$

FIG. 5.- (a) Deviations from the mean for muons in equal solid angle bins on the sky. Superposed is the best-fitting Gaussian. (b) Deviations from the mean for

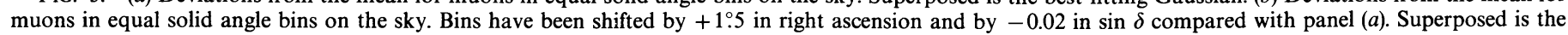
best-fitting Gaussian. 
random fluctuations. The largest deviation in this distribution is $+3.82 \sigma$; there is an a priori probability of 0.26 of finding a deviation at least this large from random fluctuations in the background.

The survey was then repeated using a set of bins shifted with respect to those of the first survey. This second survey reduces the possibility that a weak source was missed because it was located near the edge of a bin and the signal was spread into adjacent bins. For this second survey we shifted the right ascension bins by +1.5 and the $\sin \delta$ bins by -0.02 . At the pole we constructed a cap of solid angle $\Delta \Omega=2.1 \times 10^{-3}$. The remaining polar solid angle region was divided equally into 59 bins. This procedure prevents the polar bins from becoming long and narrow. As above we computed the deviation from the mean for every solid angle bin. Figure $5 b$ shows the distribution of these deviations for the 4020 bins in which the number of events is $\geq 16$ and $\sin \delta \geq-0.34$. Superposed onto this distribution is the best-fitting Gaussian, for which $\chi^{2} /$ DoF $=71.0 / 70$. The mean of the Gaussian is $-3.8 \times 10^{-3}$ and $\sigma$ is 1.02 , as expected for a distribution of random fluctuations. The largest deviation in this distribution is $+3.97 \sigma$; it does not overlap the largest deviation in the unshifted survey. There is an a priori probability of 0.14 of finding a deviation this large from random fluctuations in the background.

We conclude that there is no evidence for a steady source of muon emission in our data.

Although there are apparently no muon point sources present in our data, we have nevertheless investigated whether any high-energy sources are found within the solid angle bins with the largest deviations $(>3.5 \sigma)$. There are no coincidences in the catalogs of Joss \& Rappaport (1984) and Nagase (1989). There is an X-ray source with no optical counterpart, $1 \mathrm{H}$ $1151-130=4 \mathrm{U} 1147-12$ (Woods et al. 1984), within the bin with the largest deviation in the unshifted survey.

In Figure 6 MACRO's exposure $A_{\text {eff }} \times f \times t_{\text {expos }}$ is plotted as a function of $\sin \delta$. The average effective area, $A_{\text {eff }}$, for each bin was computed by averaging the projected area seen by each muon, $A_{\text {eff }}=\sum_{i} A\left(\theta_{i}, \phi_{i}\right) / n_{\text {obs }}$, where $\theta_{i}, \phi_{i}$ are the angular coordinates of the muon in the detector frame and the expres-

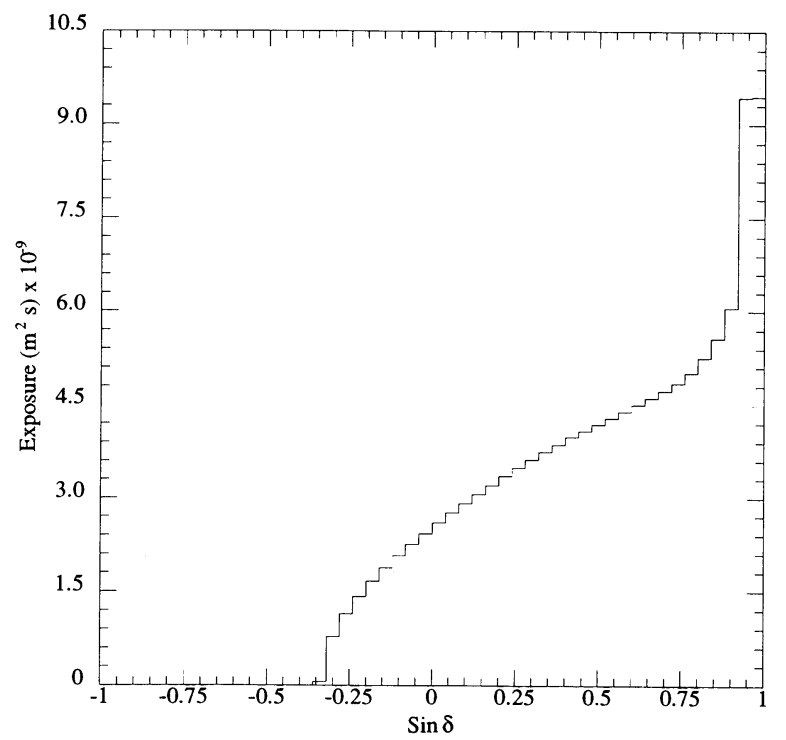

FIG. 6.-Average exposure of MACRO as a function of $\sin \delta$, computed as described in the text. sion for $A\left(\theta_{i}, \phi_{i}\right)$ assumes that the detector consists of 10 horizontal planes of streamer tubes, at least four of which must be hit (Bologna et al. 1985). The fractional exposure time, $f$, was computed by checking which bins were $\leq 72^{\circ}$ from the zenith at the arrival of each event. The total exposure time, $t_{\text {expos }}$ was taken from Table 2 .

Following the prescription of Helene (1983), we have used $n_{\text {obs }}$ and $n_{\text {exp }}$ to compute the upper limit with $95 \%$ C.L. for the undetected muon signal in each bin, $n_{\mu}(95 \%)$. The upper limit with $95 \%$ C.L. to the muon flux in each bin, $J_{\mu}^{\text {stdy }}(95 \%)$, was computed from

$$
J_{\mu}^{\text {stdy }}(95 \%) \leq \frac{n_{\mu}(95 \%)}{\bar{\epsilon} A_{\text {eff }} f t_{\text {expos }}} \mathrm{cm}^{-2} \mathrm{~s}^{-1},
$$

where $\bar{\epsilon}$ is the average efficiency for each solid angle bin. Explicit computation shows that the average efficiency has little dependence on right ascension and declination, and is approximately given by $\bar{\epsilon} \approx 0.81 \times 0.81$, where the first factor is due to bin size $(\S 2)$ and the second factor is due to the efficiency of track reconstruction $(\S 3)$.

The $95 \%$ confidence limits to the muon flux, $J_{\mu}^{\text {stdy }}(95 \%)$, for all bins are shown in Figure 7 as gray-scale intensity levels mapped in equatorial coordinates. A typical upper limit at $\delta \sim 45^{\circ}$ is of order $J_{\mu}^{\text {stdy }}(95 \%) \lesssim 2 \times 10^{-12} \mathrm{~cm}^{-2} \mathrm{~s}^{-1}$.

\section{PERIODICITY SEARCH FOR SELECTED POINT SOURCES}

It is well-known that when the emission from a source is modulated with an a priori known period and a small duty cycle, the signal-to-noise ratio can be improved by a periodicity analysis. In fact the reports of a statistically significant muon excess in the X-ray binary system Cyg X-3 (Marshak et al. 1985a, b; Battistoni et al. 1985) at a particular phase of the orbital period were the result of such an analysis. In the following we describe the results of periodicity analyses of the muons from the direction of Cyg X-3 and several other sources with well-established periods. The sources investigated are listed in Table 3 (except for 1E $2259+59$ which was only included in the burst analysis). Included in Table 3 for each source are the position in equatorial coordinates; the fractional time, $f$, the source is $\leq 72^{\circ}$ from the zenith; the average effective area, $A_{\text {eff }}$; and $\langle$ depth $\rangle$, the average pathlength traversed by muons from the source direction in m.w.e.

For the muons with directions pointing back to a 1.5 halfangle cone centered on Cyg X-3, we have constructed a phase diagram based on an extrapolation of the quadratic ephemeris that van der Klis \& Bonnet-Bidaud (1989) fitted to the X-ray light curve obtained by EXOSAT. The arrival time of each muon was first corrected for the Earth's motion with respect to the solar system barycenter, and then was folded with the extrapolated light curve to obtain the phase. The phase

TABLE 3

POINT SOURCES INVESTIGATED

\begin{tabular}{|c|c|c|c|c|c|}
\hline Source & $\alpha(1950)$ & $\delta(1950)$ & $f$ & $\begin{array}{c}A_{\text {eff }} \\
\left(\mathrm{m}^{2}\right)\end{array}$ & $\begin{array}{l}\langle\text { depth }\rangle \\
\text { (m.w.e.) }\end{array}$ \\
\hline 4U $0115+63$ & $1^{\mathrm{h}} 16^{\mathrm{m}}$ & $63^{\circ} 33^{\prime}$ & 0.864 & 213.0 & 3731 \\
\hline Crab ........... & 532 & 2159 & 0.467 & 227.3 & 3608 \\
\hline Her $\mathrm{X}-1 \ldots \ldots$ & 1656 & 3525 & 0.550 & 223.0 & 3902 \\
\hline $4 U 1907+09 \ldots \ldots$ & 1909 & 940 & 0.421 & 213.1 & 3556 \\
\hline Cyg X-1 ......... & 1956 & 3503 & 0.549 & 220.5 & 3914 \\
\hline Cyg X-3 …...... & 2030 & 4045 & 0.582 & 216.4 & 4008 \\
\hline $1 \mathrm{E} 2259+59 \ldots \ldots$ & 2259 & 5831 & 0.747 & 207.6 & 3852 \\
\hline
\end{tabular}




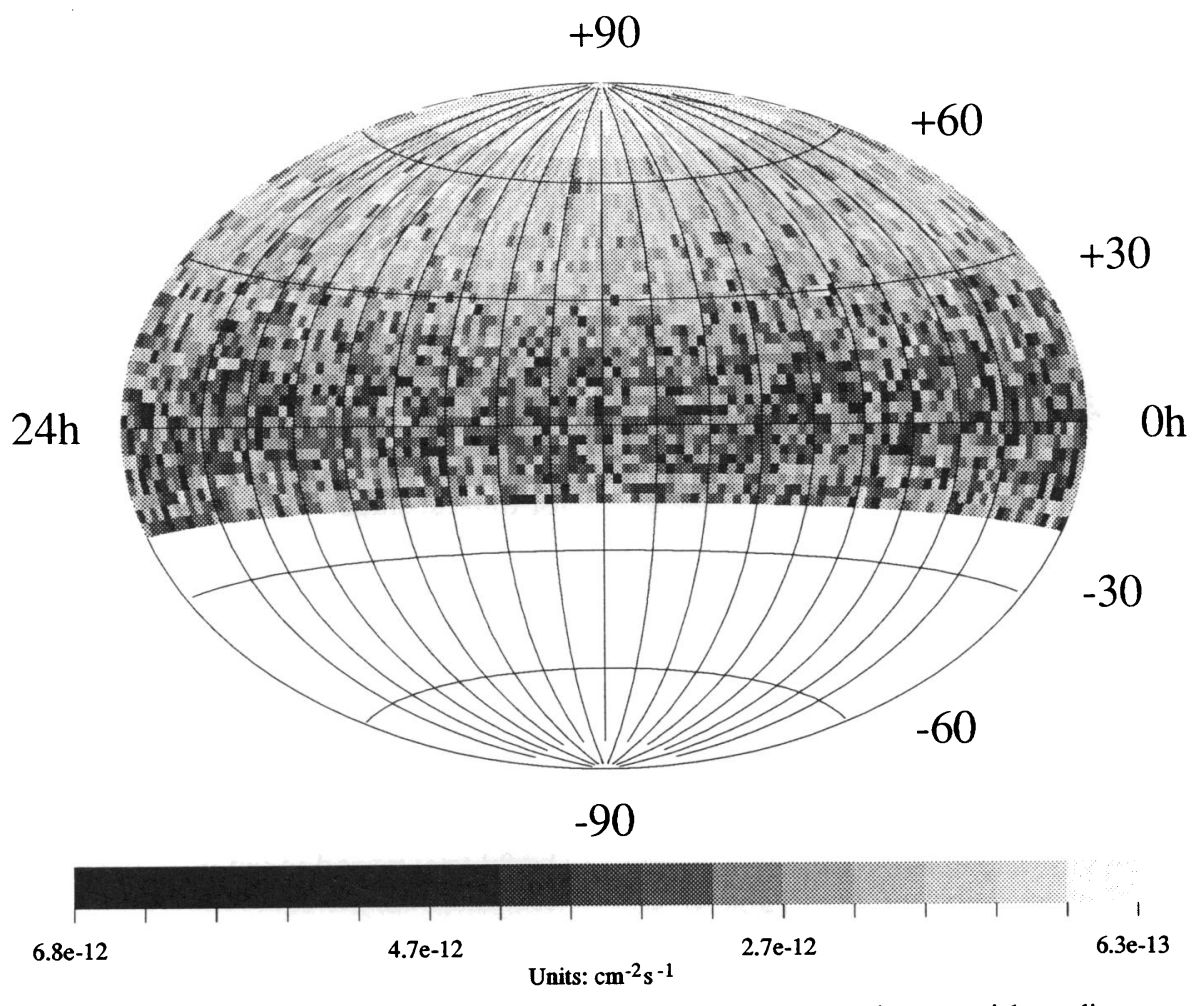

FIG. 7.-The $95 \%$ confidence limits on the muon flux for the all-sky survey in equatorial coordinates

diagram for two complete cycles is shown in Figure 8. The average expected number of muons in each phase bin is also shown in Figure 8 as a dashed line. From this figure we see that the largest deviation in one complete cycle is found in phase bin $0.8 \leq \Phi \leq 0.9$ and its magnitude is $\approx 2.1 \sigma$. We therefore conclude that our data do not show evidence for a modulated muon signal with this period.

We have computed the $95 \%$ confidence limit to the modulated muon flux from the direction of Cyg X-3 using an equa-

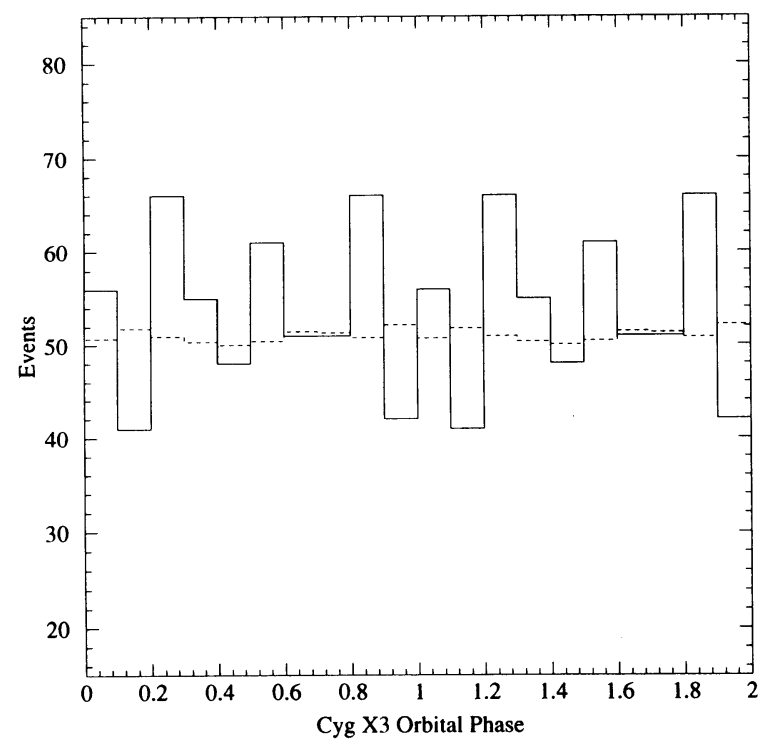

FIG. 8.-Phase diagram for muon events from a $1: 5$ half-angle cone centered on Cyg X-3. The period for the phase computation comes from the parabolic ephemeris of van der Klis \& Bonnet-Bidaud (1989). tion analogous to equation (1). Since the modulated muon emission reported by Soudan 1 (Marshak et al. 1985a, b) and NUSEX (Battistoni et al. 1985) was found in a phase bin of width $\Delta \Phi=0.1$, we have used in our computation the phase bin which shows the largest fluctuation, $0.8 \leq \Phi \leq 0.9$. In Table 4 and Figure 9 we give this $95 \%$ confidence limit. On Figure 9 we have in addition displayed the limits and observations reported by other underground experiments.

In Table 4 we also give the results of periodicity analyses on several additional X-ray binaries extracted from the compilations of Joss \& Rappaport (1984) and Nagase (1989). These sources all have well-determined ephemerides and stable periods (see references in Table 4).

We have also searched for a periodic muon signal from the Crab pulsar. The Crab pulsar is a pulsed X-ray emitter and the Crab nebula is a known $\mathrm{TeV} \gamma$-ray source. Although its pulsar

TABLE 4

Search for Modulated Muon Signals from Point Sources

\begin{tabular}{|c|c|c|c|c|}
\hline Source & $P_{0}$ & Type & $W\left(\geq Z^{2}\right)$ & $\begin{array}{c}J_{\mu}^{\bmod }(95 \%) \\
\left(\mathrm{cm}^{-2} \mathrm{~s}^{-1}\right)\end{array}$ \\
\hline $4 \mathrm{U} 0115+63 \ldots \ldots$ & 24.32 days $^{\mathrm{a}}$ & Orbital & 0.79 & $<8.9 \times 10^{-13}$ \\
\hline Crab .............. & $33.3 \mathrm{~ms}^{\mathrm{b}}$ & Pulsar & 0.90 & $<1.1 \times 10^{-12}$ \\
\hline Her X-1 ........... & 1.70 days $^{c}$ & Orbital & 0.98 & $<9.4 \times 10^{-13}$ \\
\hline $4 \mathrm{U} 1907+09$ & 8.38 days $^{d}$ & Orbital & 0.57 & $<1.4 \times 10^{-12}$ \\
\hline Cyg X-1 …...... & 5.60 days $^{e}$ & Orbital & 0.68 & $<9.0 \times 10^{-13}$ \\
\hline Cyg X-3 ............... & $4.79 \mathrm{hr}^{\mathrm{f}}$ & Orbital & 0.06 & $<1.1 \times 10^{-12}$ \\
\hline
\end{tabular}

a Ricketts et al. 1981

b Massaro et al. 1991.

c Ögelman 1987.

d Bolton 1975.

e Makishima et al. 1984.

f van der Klis \& Bonnet-Bidaud 1989. 


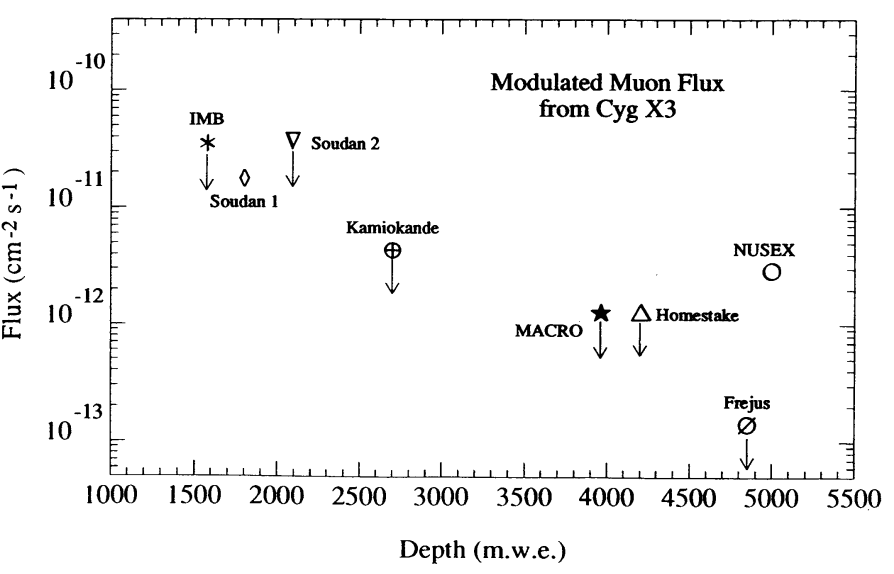

Fig. 9.-MACRO limit to the modulated muon flux from Cyg X-3 compared with the results from other underground detectors.

period is extremely short, $33 \mathrm{~ms}$, the Crab pulsar is nevertheless an extremely accurate clock with a period determined to better than 10 significant figures (Massaro et al. 1991) and a periodicity analysis would find a signal if one were present.

The results of all these searches were negative, and the corresponding flux limits, $J_{\mu}^{\text {mod }}(95 \%)$, are also reported in Table 4.

\section{SEARCH THROUGH PERIOD SPACE}

Since the ephemerides we have used in our periodicity analyses are derived from measurements that are typically several years old, there is a reasonable probability that the uncertainty in the period, when extrapolated to the time of our observations, will have washed out the signal. This can be seen by simple error analysis. The error in the phase, $\delta \Phi$, accumulates over the length of the observation period, $T$, as

$$
\delta \Phi \approx \frac{T}{P^{2}} \delta P=N \frac{\delta P}{P},
$$

where $P$ is the period, $\delta P$ is the error in the period, and $N=T / P$ is the number of periods. Therefore, a successful periodicity analysis requires $\delta P / P \ll N^{-1}$. For our analysis of Cyg X-3, which uses data obtained over 2 years, its period must be known with a relative error of $\sim 0.02 \%$. Since the ephemeris of van der Klis \& Bonnet-Bidaud (1989) is based on the X-ray light curve of 1983-1985, our extrapolation may not have the required precision.

We have therefore searched through period space for a modulated signal from the sources in Table 4 with a period displaced from the fiducial $\mathrm{X}$-ray period $\boldsymbol{P}_{\mathbf{0}}$. For this analysis we divided the data into four samples with approximately equal run times. Two of the samples are coincident with data sets 1 and 2 while samples 3 and 4 result from the division of data set 3 equally into two. We investigated these four data samples of approximately equal run time so that each sample would have approximately the same independent Fourier spacing (IFS), $\delta P=P^{2} / \Delta t$, where $\Delta t$ is the total length of time over which the data were obtained (see Table 1). The IFS is the standard increment used in period searches.

In order to evaluate the statistical significance of a potential signal, we use the $Z^{2}$ statistic (Buccheri \& Sacco 1985) given by

$$
Z^{2}=\frac{1}{n_{\text {obs }}} \sum_{j=1}^{5}\left\{\left[\sum_{i=1}^{n_{\mathrm{obs}}} \cos \frac{\left(2 \pi j t_{i}\right)}{P}\right]^{2}+\left[\sum_{i=1}^{n_{\mathrm{obs}}} \sin \frac{\left(2 \pi j t_{i}\right)}{P}\right]^{2}\right\},
$$

where $t_{i}$ is the arrival time of the $i$ th event, $P$ is the running trial period, and $n_{\text {obs }}$ is as above the total number of muons in the window. This statistic, which is the sum of the Rayleigh powers of the first five harmonics (de Jager 1987), is appropriate because the modulated muon emission reported for Cyg X-3 had a relatively small duty cycle $(\sim 10 \%)$ in the phase diagram. It follows from the Shannon sampling theorem that the sum of five harmonics of the basic periodicity is needed to reconstruct the signal (Wiener 1964).

The a priori distribution of the $Z^{2}$ statistic is a $\chi^{2}$ distribution with 10 d.o.f. (Buccheri \& Sacco 1985), assuming there are no gaps in data taking. This is not our case. Therefore, we evaluated the significance of the deviations observed for the test statistic by constructing artificial data sets in which the events were distributed randomly in phase.

In Figure 10 we show the results of our search through period space for a modulated muon signal from Cyg X-3 in the four samples, plotting the value calculated for $Z^{2}$ as a function of the trial period. The dotted lines represent the estimated $1 \%$ probability levels from the Monte Carlo calculations. In Table 4 we give our estimate of the confidence level of the null hypothesis $W\left(\geq Z^{2}\right)$, namely the probability of finding by chance a random fluctuation of the statistics as large as $Z^{2}$, for the fiducial period taken from the ephemerides. This statistical test reinforces the conclusion that there is no modulated signal present at the fiducial period.

It can be seen in Figure 10 that there is no large fluctuation which appears consistently at the same period in the four samples. Nevertheless there are fluctuations with a probability $\leq 1 \%$. To estimate the significance level of fluctuations, $W\left(\geq Z^{2}[\max ]\right)$, we must make a correction for the number of trials, $N$. For this investigation $N=101 \times 4=404$ because we have searched through 101 IFS in four independent runs. Let $p$ be the probability of an occurrence of a fluctuation as large as $Z^{2}(\max )$, as determined by Monte Carlo methods. Then $W\left(\geq Z^{2}[\max ]\right)=1-(1-p)^{N}$. With this correction, $W\left(\geq Z^{2}[\max ]\right) \simeq 1$. The result is that we have no evidence of a modulated muon signal from Cyg X-3, with a period displaced from the nominal $X$-ray period by $\lesssim 5 \%$.

The same search through period space was applied to Her X-1, 4U 1907+09, and Cyg X-1. As for Cyg X-3, we divided the data into four samples with approximately equal run times, and we used the IFS for data set 2 . We have investigated 50 IFS on either side of the fiducial periods in steps of 1 IFS for a total of 101 possible periods. For these sources, as for Cyg $\mathrm{X}-3, W\left(\geq Z^{2}[\max ]\right) \simeq 1$.

\section{SEARCH FOR BURSTING EPISODES}

One of the most striking peculiarities of the Cyg X-3 system is its episodes of flaring activity at radio wavelengths. On the average, once or twice per year the radio emission from this object, which is ordinarily at a quiescent flux density of a few $\mathrm{mJy}$, rapidly jumps to a flux density of tens of Jy and remains there for a few days. Recently Thomson et al. (1991) reported the detection of two unmodulated muon bursts from Cyg X-3 correlated with a large radio outburst that occurred in 1991 January. The radio burst peaked on 1991 January 21, and the two unmodulated muon excesses that were observed on 1991 January 20 and 23 had fluxes $J_{\mu} \simeq 7.5 \times 10^{-10} \mathrm{~cm}^{-2} \mathrm{~s}^{-1}$ at 2090 m.w.e. The fact that a positive underground muon signal was reported around the time of the radio burst has led to the suggestion that the many reports of the absence of a muon 

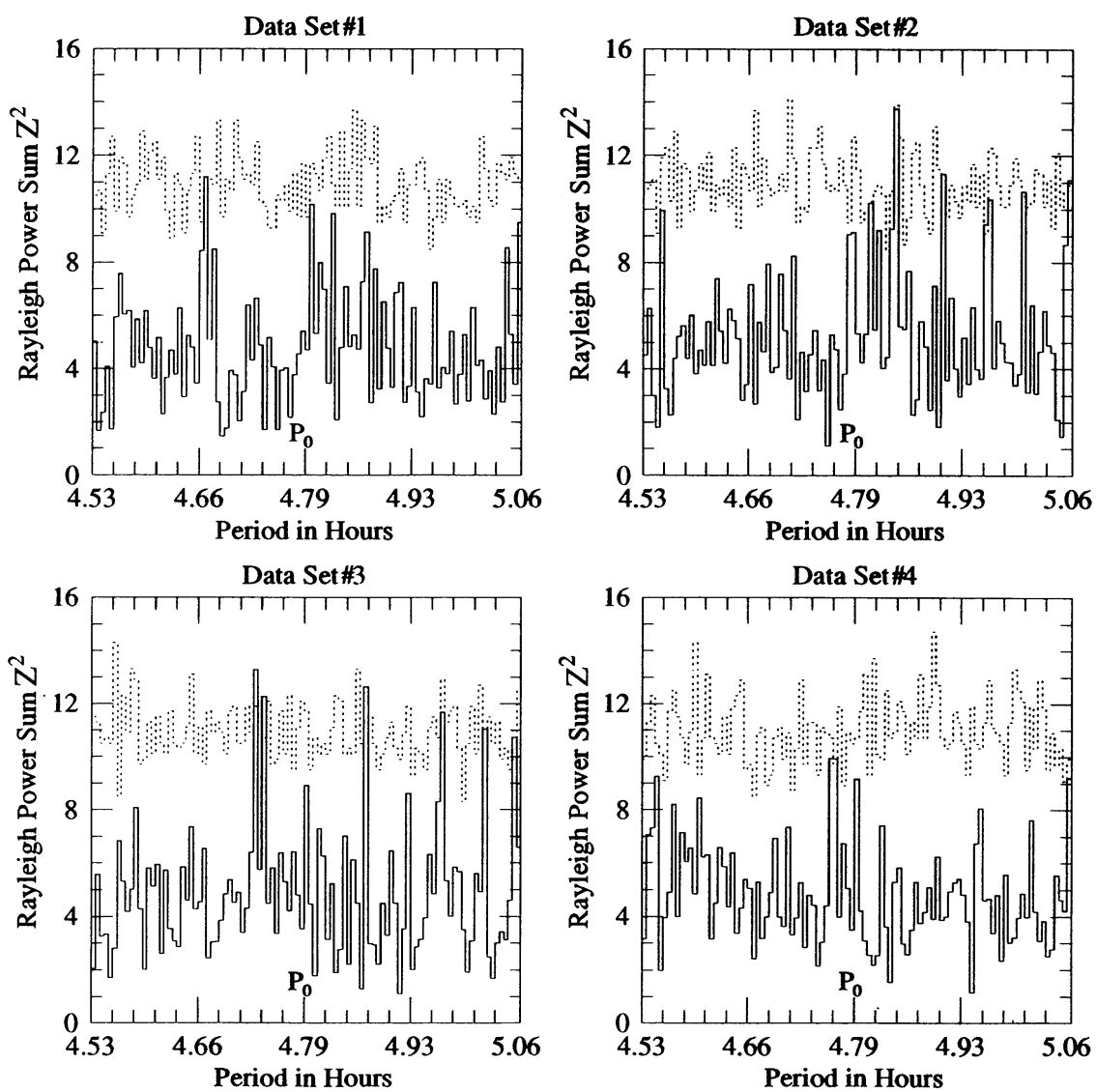

FIG. 10.- Search trhough period space for a modulated muon signal from Cyg X-3 for the four data samples. The solid lines are the $Z^{2}$ statistic; the dashed lines are $1 \%$ probability levels computed by Monte Carlo methods. $P_{0}$ is the fiducial period from the parabolic ephemeris of van der Klis $\&$ Bonnet-Bidaud (1989).

signal from Cyg X-3 might be related to its relatively quiescent state at the time the observations were made.

We have searched our complete data set, which includes data taken at the time of the 1991 January radio flare, for a statistically significant daily burst of muons from the direction of Cyg X-3. In addition, we have searched for daily bursts from the remaining six sources in Table 5. For these searches we compute the quantity $-\log _{10} \Lambda$, where

$$
\Lambda=1-\sum_{n=0}^{n_{\text {obs }}}\left(n_{\text {exp }}\right)^{n} \exp \left(-n_{\text {exp }}\right) / n !
$$

Here $n_{\mathrm{obs}}$ is the number of muons seen on any particular day and $n_{\text {exp }}$ is the expected number computed by Monte Carlo simulation. The $\Lambda$ statistic represents the probability of observing a burst at least as large as $n_{\text {obs }}$ due to fluctuations in the background, which are assumed to have a Poissonian distribution about $n_{\text {exp }}$.

In the absence of muon bursts, the a priori expectation is that the cumulative frequency distribution of $\Lambda$ is a power law of index -1 since any $\mathrm{A}$ is equally probable. This expectation, however, is only valid for observation trials of equal length. For the seven sources in Table 5 we have computed the cumulative frequency distribution of $\Lambda$ for the 135 days in which the detector had less than $1 \%$ dow.time. In Figure 11 we show a $\log -\log$ plot of this cumulative frequency distribution. The straight line resulting from a leasi-squares fit to this distribution has a slope of -0.97 and is shown superposed. Since this straight line fits the data well, we conclude there are no statistically significant daily bursts in this data set.

The result of our search for a statistically significant burst from the direction of Cyg X-3 for all days in the three data sets, including days with significant downtime, is shown in Figure 12. In this figure we have also marked the dates of all reported radio bursts during the period MACRO was operational (G. Umana 1991, private communication). In Figure 12 there are 7 days in which $-\log _{10} \Lambda \geq 2.5$, and 2 days in which $-\log _{10} \Lambda \geq 3.0$. We have tested whether the number of muons seen on these days represent statistically significant daily bursts by simulating the daily background in the direction of Cyg X-3 using Poissonian statistics and $n_{\text {exp }}$. The results of 1000 simulations show that the chance probability of observ-

TABLE 5

SEARCH FOR BURSTING BEHAVIOR FROM POINT SOURCES

\begin{tabular}{|c|c|}
\hline Source & $\begin{array}{l}J_{\mu}^{\text {burst }}(95 \%) \\
\left(\mathrm{cm}^{-2} \mathrm{~s}^{-1}\right)\end{array}$ \\
\hline $4 U 0115+63$ & $<9.4 \times 10^{-11}$ \\
\hline Crab............. & $<2.4 \times 10^{-10}$ \\
\hline Her X-1 $\ldots \ldots \ldots \ldots \ldots$ & $<1.5 \times 10^{-10}$ \\
\hline $4 U 1907+09 \ldots \ldots \ldots$. & $<2.2 \times 10^{-10}$ \\
\hline Cyg X-1 $\ldots \ldots \ldots \ldots \ldots$ & $<1.5 \times 10^{-10}$ \\
\hline Cyg X-3 .............. & $<2.0 \times 10^{-10}$ \\
\hline $1 E 2259+59 \ldots \ldots \ldots$ & $<1.5 \times 10^{-10}$ \\
\hline
\end{tabular}




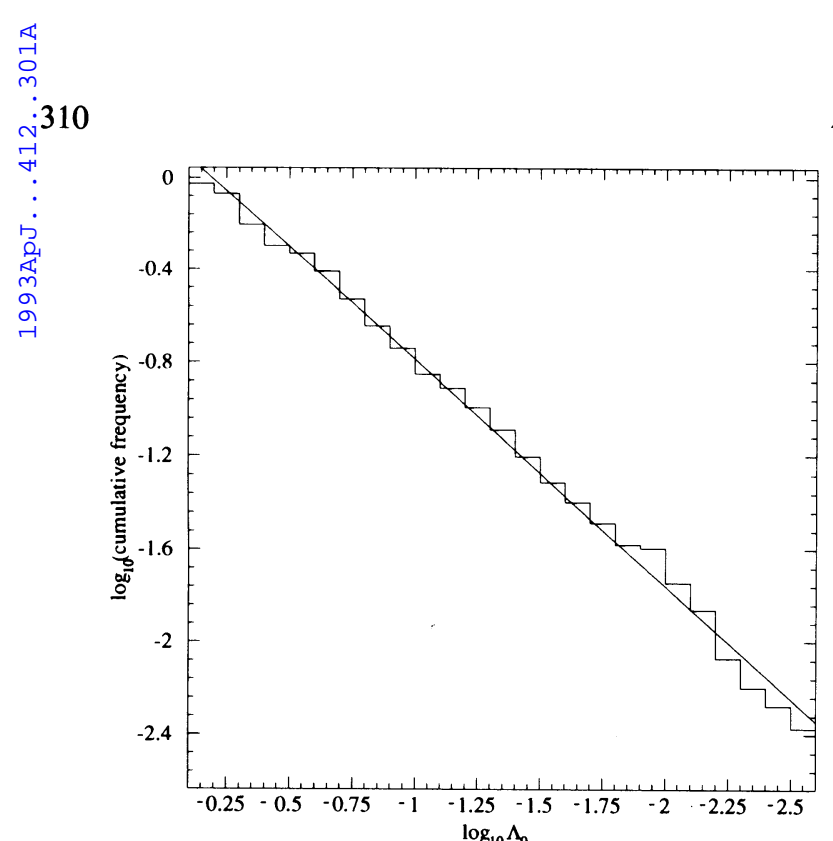

FIG. 11.-The log of the cumulative frequency distribution vs. $\log _{10} \Lambda \geq$ $\log _{10} \Lambda_{0}$ for 945 source-days for the sources listed in Table 5. Superposed is a least-squares fit straight line whose slope is -0.97 .

ing seven fluctuations with $-\log _{10} \mathrm{~A} \geq 3.0$ is $43 \%$. We conclude that MACRO has not observed a statistically significant daily burst from the direction of Cyg X-3. In Table 5 we give the upper limit with $95 \%$ C.L. to the flux, $J_{\mu}^{\text {burst }}(95 \%)$, for the largest daily fluctuation in Figure 12.

We have also searched for a daily, unmodulated muon excess from the remaining six sources in Table 5 . As for Cyg X3 we find no evidence for a statistically significant daily fluctuation during any of the approximately 500 days each source was observed. For each source we give the upper limit with $95 \%$ C.L. to the flux, $J_{\mu}^{\text {burst }}(95 \%)$, for the day in which the largest daily fluctuation was found.

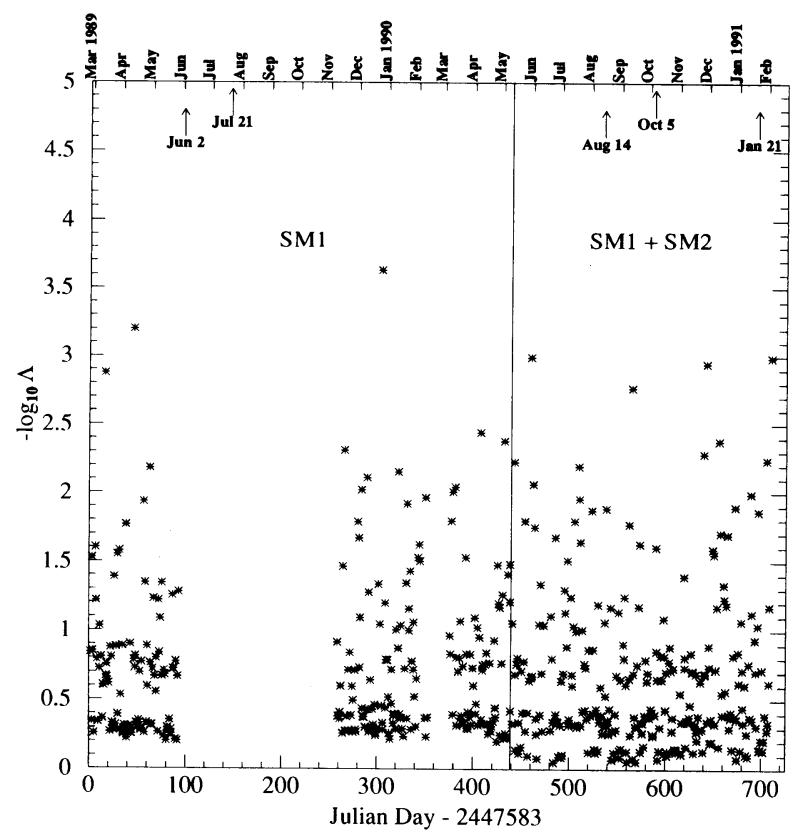

FIG. 12.-The search for daily, unmodulated muon excesses from Cyg X-3. Plotted is $-\log _{10} \Lambda$ as a function of Julian day. The figure has been divided at the date on which both SM1 and SM2 became operational. The dates of several radio bursts are marked.

\section{SOURCE PARAMETERS FOR CYG X-3}

Up to this point we have computed detector-dependent flux limits for sources. However, by assuming a physical model, we can calculate detector-independent source parameters. We have derived upper limits to the Cyg X-3 source parameters assuming an integral spectrum of the form $K_{s}\left(\alpha_{s}\right) E_{\mu}^{-\alpha_{s}}$. With this spectral form, the number of muons observed at MACRO is

$$
\begin{aligned}
& n_{\mu}=\left(-\alpha_{s}\right) \int_{\text {run-time }} d t \int_{E_{\mu} \min }^{\infty} d E_{\mu} K_{s}\left(\alpha_{s}\right) E_{\mu}^{-\left(\alpha_{s}+1\right)} \\
& \times A_{\text {eff }}(\theta, \phi, t) \epsilon(t) g(\cos \theta) P\left(E_{\mu}, h\right) .
\end{aligned}
$$

Here $A_{\text {eff }}(\theta, \phi, t)=A_{\text {eff }}[\theta(t), \phi(t), t]$ is the detector effective area computed as described for Figure 5. The zenith angle, $\theta=\theta(t)$ and the azimuthal angle $\phi=\phi(t)$ are functions of time because of the changing position of Cyg X-3 with respect to MACRO; the explicit time dependence in $A_{\text {eff }}$ is the result of the changing number of supermodules used in the data collection. In addition, $\epsilon=\epsilon(t)$ is the time-dependent, run-by-run detector efficiency; $g(\cos \theta)=\sec \theta$ is the dependence of muon production on zenith angle, assumed the same as high-energy muon production in the atmosphere (Gaisser 1990); and $P\left(E_{\mu}, h\right)$ is the survival probability for muons of initial energy $E_{\mu}$ to penetrate a slant-depth $h$. Again because of the changing position of Cyg X-3 with respect to MACRO, $h=h(t)$.

We have computed upper limits to the source parameters in Cyg X-3 from equation (4) using our $95 \%$ confidence limits to the steady number of muons (119.8), the modulated number of muons (21.9), and the average daily number of muons (4.0) seen during data set 3 . For $P\left(E_{\mu}, h\right)$, we used the survival probabilities calculated by Bilokon et al. (1992) who propagated muons through the average composition of Gran Sasso rock using GEANT (Brun et al. 1991). For this computation, we assumed

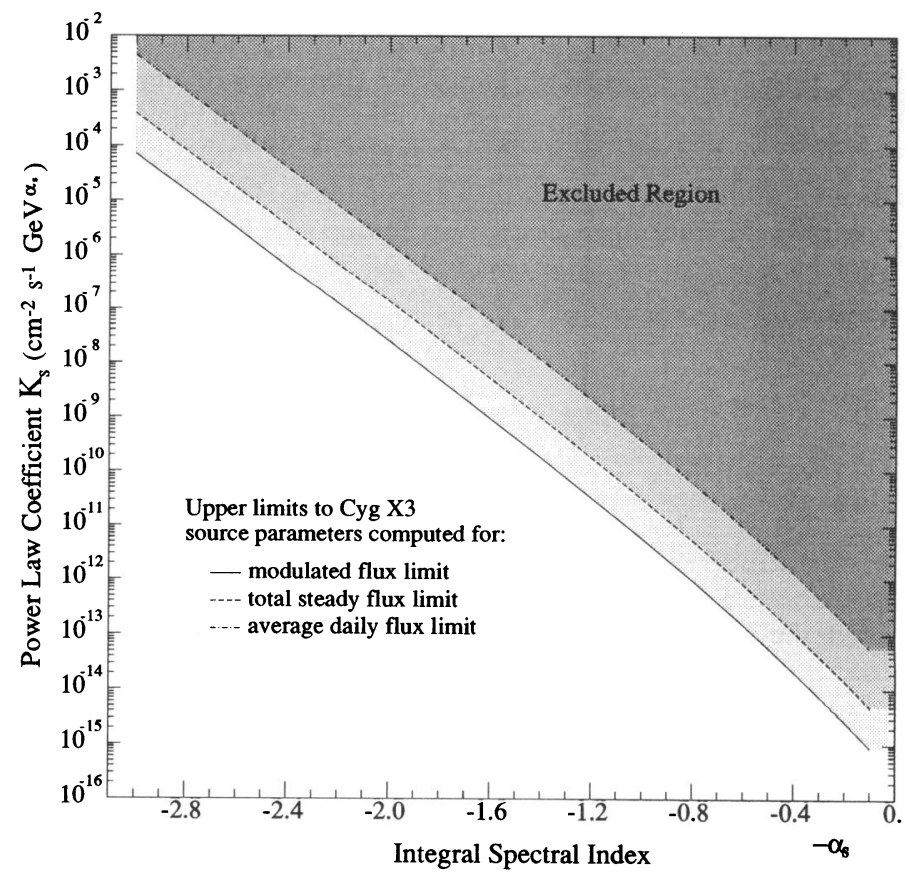

FIG. 13.-Upper limits to the detector-independent source parameters for Cyg X-3 for a range of spectral indices, as computed from equation (4). The upper limits have been computed for the $95 \%$ confidence limits to the steady, modulated, and average daily muon flux. 
that $81 \%$ of the muons from Cyg X-3 that survive to the detector fall within the 1.5 search window The integral was evaluated by Monte Carlo methods, and the results are shown in Figure 13.

We have repeated the above computations for a source model which assumes prompt muon production, that is, $g(\cos \theta)=1$. For this model, the upper limits are a factor of $\sim 1.2$ above those shown in Figure 13 for $\alpha_{s}=-2.0$. This factor, quantifying the difference between the two source models, varies by less than $10 \%$ over the entire range of spectral indices investigated.

\section{CONCLUSIONS}

We have investigated approximately $1.8 \times 10^{6}$ muons collected by the first and second MACRO supermodules during a little more than a year of live time. Our all-sky survey for muon point sources has yielded no evidence for a source of steady muon flux. In regions with the greatest exposure, we have reached a flux limit of $F_{\text {stdy }}=7 \times 10^{-13} \mathrm{~cm}^{-2} \mathrm{~s}^{-1}$. We have searched for a periodic muon signal from Cyg X-3. We find no evidence for modulated muon emission at the fiducial X-ray period, nor at periods displaced by $\leq 5 \%$ from the fiducial. A search for daily bursts of unmodulated muon emission from Cyg X-3 has also yielded no evidence for statistically significant fluctuations. We have used our upper limits to the Cyg X3 muon flux to compute detector-independent source parameters, assuming a power-law energy spectrum of muon emission.

We have investigated several additional high-energy sources which are listed in Table 3. For these sources we find no evidence for modulated muon emission at the reported fiducial periods; searching through period space also gives negative results. Analysis of the daily muon emission from these sources shows that the fluctuations observed are consistent with random fluctuations in the background.
Ahlen, S. P., et al. 1992, Nucl. Phys. B, 370, 432 1993, Nucl. Istr. Meth. Res. A, 324, 337

Auriemma, G., Bilokon, H., \& Grillo, A. F. 1984, Nuovo Cimento, C7, 779 Auriemma, G., Maiani, L., \& Petrarca, S. 1985, Phys. Lett. B, 164, 179

Baillon, P., et al. 1991, Proc. 22d Internat. Cosmic-Ray Conf. (Dublin), 1, 220

Battistoni, G., et al. 1985, Phys. Lett. B, 155, 465

Baym, G., et al. 1985, Phys. Lett. B, 160, 181

Berezinsky, V. S., Ellis, J., \& Ioffe, B. 1986, Phys. Lett. B, 172, 473

Berezinsky, V.S., et al. 1988, A\&A, 189, 306

Berger, C., et al. 1986, Phys. Lett. B, 174, 118

Bilokon, H., et al. 1991, Macro. Memo 4/92

Bionta, R., et al. 1987, Phys. Rev. Lett., 36, 30

Bologna, G., et al. 1985, Nuovo Cimento C, 8, 76

Bolton, C. J. 1975, ApJ, 200, 269

Brun, R., et al. 1991, CERN DD/EE/84-1

Buccheri, R., \& Sacco, B. 1985, in Data Analysis in Astronomy, ed. L. Scarsi et al. (New York: Plenum), 15

Collins, J., \& Olness, F. 1987, Phys. Lett. B, 187, 376

Corbato, S. 1989, Ph.D. thesis, Univ. Pennsylvania

de Jager, O. C. 1987, Ph.D. thesis, Potchefstroom Univ.

Domokos, G., \& Nussinov, S. 1987, Phys. Lett. B, 187, 372

Drees, M., \& Halzen, F. 1988, Phys. Rev. Lett., 61, 275

Gaisser, T. K. 1990, Cosmic Rays and Particle Physics (Cambridge: Cambridge Univ. Press), chap. 6

Helene, O. 1983, Nucl. Instr. Meth., 212, 319

\section{REFERENCES}

Joss, P. C., \& Rappaport, S. A. 1984, ARA\&A, 22, 537

Makishima, K., et al. 1984, PASJ, 36, 679

Marshak, M. F., et al. 1985a, Phys. Rev. Lett., 54, 2079

- 1985b, Phys. Rev. Lett., 55, 1965

Massaro, E., et al. 1991, ApJ, 376, L11

Nagase, F. 1989, PASJ, 41, 1

Nagle, D. E., Gaisser, T. K., \& Protheroe, R. J. 1988, Ann. Rev. Nucl. Part. Sci., 38,609

O’Flaherty, K. S., et al. 1991, Proc. 22d Internat. Cosmic-Ray Conf. (Dublin), 1,257

Ögelman, H. 1987, A\&A, 172, 84

Oyama, Y., et al. 1986, Phys. Rev. Lett., 56, 991

Petrakis, J. 1990, Ph.D. thesis, Indiana Univ.

Punch, M., et al. 1992, Nature, 358, 477

Ricketts, M. J., et al. 1981, Space Sci. Rev., 30, 399

Ruddick, K. 1985, Phys. Rev. Lett., 54, 2079

. 1986, Phys. Rev. Lett., 57, 531

Stanev, T., Vankov, C. P., \& Halzen, F. 1985, Proc. 19 Internat. Cosmic-Ray

Conf. (La Jolla), 7, 219

Vacanti, G., et al. 1991, ApJ, 377, 467

van der Klis, M., \& Bonnet-Bidaud, J. M. 1989, A\&A, 214, 203

Weekes, T. 1991, Proc. 22d Internat. Cosmic-Ray Conf. (Dublin), 5, 59

Wiener, N. 1964 Time Series (Cambridge: MIT Press)

Wood, K. S., et al. 1984, ApJS, 56, 507

Yock, P. C. M. 1991, Proc. 22d Internat. Cosmic-Ray Conf. (Dublin), 4, 528 